\title{
Suprahepatic space as an alternative site for distal catheter insertion in pseudocyst-associated ventriculoperitoneal shunt malfunction
}

\author{
Zohreh Habibi, MD, ${ }^{1}$ Mehdi Golpayegani, MD, ${ }^{1}$ Bahar Ashjaei, MD, ${ }^{2}$ Keyvan Tayebi Meybodi, MD, ${ }^{1}$ \\ and Farideh Nejat, MD, MPH${ }^{1}$
}

\begin{abstract}
Departments of ${ }^{1}$ Neurosurgery and ${ }^{2}$ Pediatric Surgery, Children's Medical Center, Tehran University of Medical Sciences, Tehran, Iran
\end{abstract}

\begin{abstract}
OBJECTIVE Distal catheter malfunction due to pseudocyst formation or intraabdominal adhesion is a significant problem, especially in pediatric patients who have limited sites for distal catheter insertion. In this study, the authors present a series of 12 patients with intractable distal shunt malfunctions due to peritoneal pseudocyst formation who underwent distal catheter replacement in the suprahepatic space to reduce the risk of distal catheter malfunction.

METHODS Twelve consecutive patients with shunt malfunction due to pseudocyst formation who had undergone ventriculosuprahepatic shunting from 2014 to 2019 were identified. According to medical records, after primary evaluations, shunt removal, and antibiotic therapy, they underwent revision surgeries with placement of a distal catheter into the suprahepatic space.
\end{abstract}

RESULTS Nine boys and 3 girls, ranging in age from 5 months to 14 years, with one or more episodes of pseudocyst formation, underwent shunt revision with placement of a distal catheter into the suprahepatic space. After a median follow-up of 31 months, none of the patients experienced further distal malfunction.

CONCLUSIONS The suprahepatic space appears to be a safe place to secure the distal end of a ventricular catheter following pseudocyst formation, with less risk of re-adhesion. This lower risk might be attributable to the lack of omentum in the suprahepatic space.

https://thejns.org/doi/abs/10.3171/2020.3.PEDS19772

KEYWORDS abdominal pseudocyst; distal shunt malfunction; suprahepatic space; ventriculoperitoneal shunt; hydrocephalus

$\mathrm{O}$ NE of the most common methods for hydrocephalus management is ventriculoperitoneal (VP) shunt insertion, with the distal end of the shunt placed in the general peritoneal cavity. ${ }^{1}$ Shunt failure is a major adverse event in these patients, with a reported rate of $29 \%$ in the first year. ${ }^{2}$ Many cases require multiple shunt revision surgeries, with potential associated risks. Among all types of shunt failure, distal catheter malfunction associated with peritoneal pseudocyst causes substantial difficulties for patients and surgeons. Pseudocyst-associated shunt failure is reported to occur in $0.7 \%-4.5 \%$ of cases. ${ }^{3}$ Despite this relatively low incidence, management is very challenging as the rate of recurrence is high and alternative spaces for distal catheter placement may not be competent. There is no consensus on the etiology and treatment of abdominal pseudocyst; nonetheless, the condition is considered a distal catheter infection by most surgeons. The most accepted approach is a period of shunt externalization and antibiotic therapy, followed by shunt reinsertion in the peritoneum or other cavities. ${ }^{3}$ However, sometimes it is difficult to find a competent place for a distal catheter, especially in pediatric patients.

In this study, we propose the suprahepatic space as an alternative site for distal catheter reinsertion in patients who experienced pseudocyst-associated shunt failure. The suprahepatic space, as a potential site for first-time distal

ABBREVIATIONS ETV = endoscopic third ventriculostomy; $L O C=$ loss of consciousness; $N E C=$ necrotizing enterocolitis; $V A=$ ventriculoatrial; VP = ventriculoperitoneal . SUBMITTED December 24, 2019. ACCEPTED March 9, 2020. 
catheter placement, was previously suggested to carry a lower rate of abdominal complications-including pseudocyst formation, blocked lower end, intestinal obstruction, inguinoscrotal migration, and visceral perforation-compared with the general peritoneal cavity. ${ }^{4}$ In our literature review, few single cases with refractory distal malfunction due to severe peritoneal adhesion were found in which the distal catheter was placed in the suprahepatic space as the only adhesion-free location. ${ }^{5}$

In this retrospective study, a series of 12 patients with pseudocyst-associated shunt failure are reported in whom shunt revision was performed with placement of a distal catheter into the suprahepatic space. The primary aim of the study is to assess the effectiveness of this alternative space by estimating the total rate of complications and pseudocyst recurrence.

\section{Methods}

This study is a retrospective consecutive case series conducted in the pediatric neurosurgery section of Children's Medical Center in Tehran. This study was approved by our Institutional Sciences Ethical Committee.

\section{Patients and Clinical Data}

A series of 12 pediatric patients who underwent ventriculosuprahepatic shunting were retrospectively identified and their medical records were reviewed. We included all patients younger than 18 years of age with pseudocystassociated shunt malfunction who underwent distal catheter repositioning in the suprahepatic space between June 2014 and May 2019. Forty-three patients with peritoneal pseudocysts were managed in our center within this period, from whom 12 cases $(27.9 \%)$ were ultimately selected for suprahepatic shunting. All patients were evaluated with brain CT, CSF analysis and culture, shunt series, and then abdominal ultrasonography or CT. Temporary CSF diversion was performed, followed by appropriate antibiotic administration. CSF samples were taken every other day during temporary diversion and the obtained samples were cultured for 72 hours. Patients with positive culture received antibiotics based on an antibiogram and those with negative CSF culture were administrated empirical antibiotics for at least 2 weeks. A new shunting procedure was not performed unless the patient had at least 3 consecutive negative CSF cultures and the pseudocyst showed resolution on serial ultrasonography.

At our institution, in patients who are not good candidates for endoscopic third ventriculostomy (ETV), the preferred diversion system after any distal infection or pseudocyst formation is a new VP shunt in the other side of the peritoneum. If the condition recurs, the next shunt is planned to be inserted in one of the available extraperitoneal spaces. Those who are estimated to have no suitable extraperitoneal space because of low body weight and small body mass, or congenital heart disease, are chosen for suprahepatic shunting. Infants are not generally considered good candidates for atrial or pleural catheters because of lower capacity of fluid absorption of these extraperitoneal spaces in this age group. In some situations, the plan would be abruptly changed to suprahepatic positioning during the first VP shunt revision due to encountering severe peritoneal adhesions that cannot be released despite meticulous dissection. Moreover, those with failed extraperitoneal shunting are chosen for suprahepatic catheter placement. A diagram depicting different plans for CSF diversion systems after pseudocyst resorption is presented in Fig. 1.

\section{Surgical Procedure}

With the patient under general anesthesia and supine, the previous right subcostal incision was slightly extended toward the right upper quadrant. Tunneling and connection to the proximal catheter was performed as routine. The ventricular system was generally entered via an occipital approach, and medium-pressure valves were routinely used. A laparotomy was created by a pediatric surgeon (B.A.) and the suprahepatic space was approached. Despite massive adhesions and fibrotic bands in the general peritoneal cavity, the suprahepatic space had remained adhesion-free in all cases. Under direct vision, the tip of the distal catheter was gently slipped over the glossy surface of the liver below the right hemidiaphragm (Fig. 2). After securing the catheter in place and suturing the peritoneum, the wound was closed in a standard fashion.

\section{Surveillance}

During the first few weeks after the surgery, the patients were closely observed for signs of shunt malfunction. Afterwards, regular outpatient surveillance was performed on a monthly basis for the first 6 months and then with longer intervals. A control abdominal ultrasound was performed several weeks after surgery to rule out new pseudocyst formation.

\section{Results}

Between June 2014 and May 2019, 43 patients with peritoneal pseudocysts were managed in our institute via shunt removal, temporary CSF diversion, serial CSF cultures, and appropriate antibiotic therapy, followed by a new permanent CSF diversion surgery. Twelve patients (9 boys and 3 girls), ranging in age from 5 months to 14 years, were retrospectively identified to have undergone suprahepatic catheter positioning. Those who underwent suprahepatic shunting consisted of 4 infants with very low weight and small bodies, a 24-month-old underweight boy with very poor general condition after recovering from severe sepsis, a 9-year-old girl with cyanotic heart disease and cor pulmonale, and 3 cases with failed extraperitoneal systems. In 4 cases (including 1 of the infants), the plan was directly shifted toward suprahepatic reposition during the first VP revision after the resorption of the pseudocyst, because the surgical team encountered severe adhesions that could not be released by intraabdominal dissection (Table 1).

The underlying diseases included congenital hydrocephalus, posthemorrhagic hydrocephalus, and postinfectious hydrocephalus (Table 1). Eight patients had more than 1 episode of pseudocyst-associated shunt failure. The average number of prior shunt revisions was $3.33 \pm 1.66$ per patient. Three patients had a history of failed ventriculoatrial (VA) or ventriculopleural shunts. The CSF or catheter culture at the time of pseudocyst formation was 


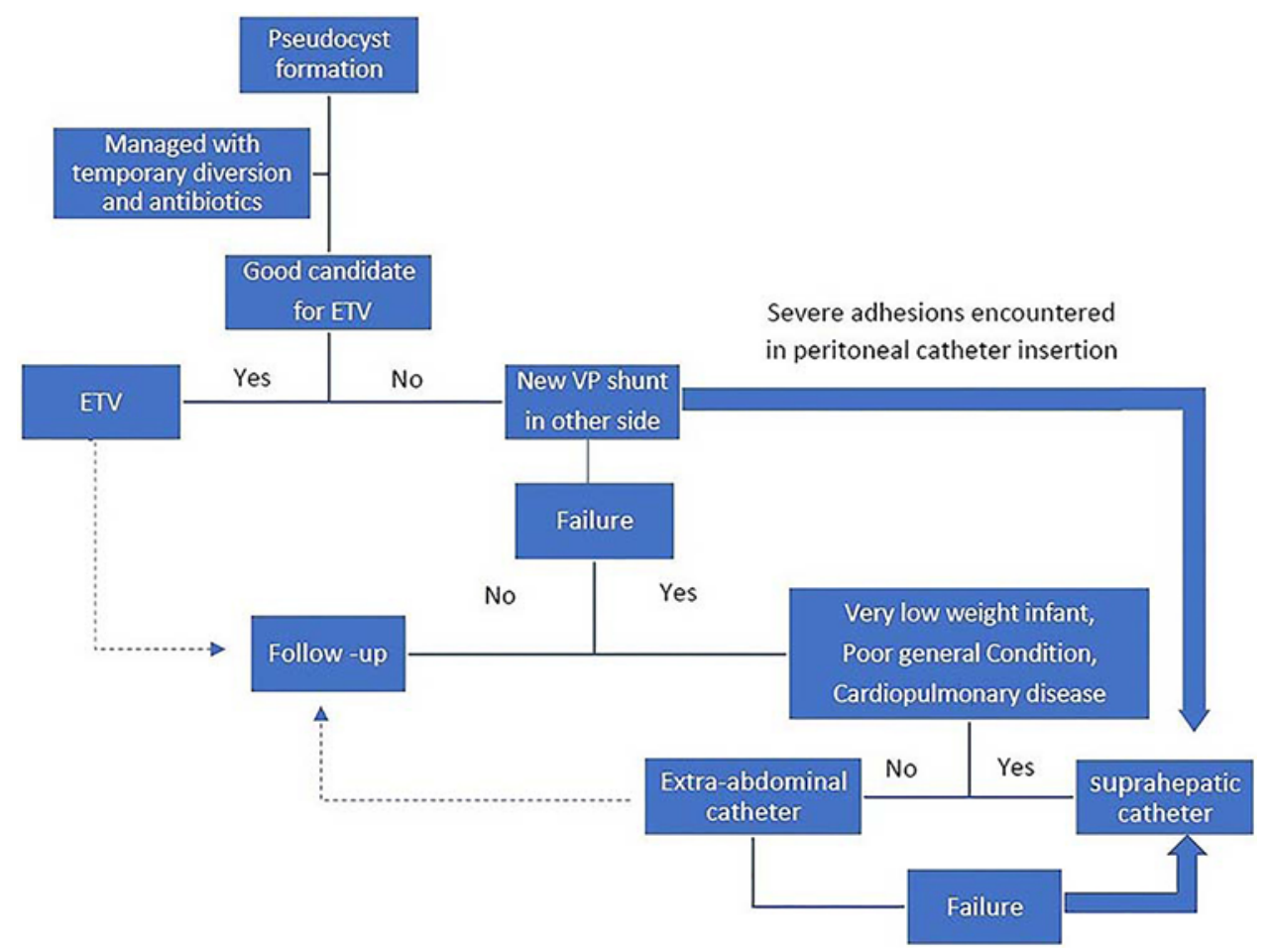

FIG. 1. The algorithm for the selection of a permanent CSF diversion system after pseudocyst formation. Figure is available in color online only.

positive in 3 cases (Pseudomonas, Acinetobacter, and Candida). Two patients had a history of previous shunt infection and 1 patient had experienced necrotizing enterocolitis (NEC) and intestinal perforation. The presenting symptoms of distal shunt malfunction included abdominal pain and distension, CSF collection around the catheter and pump, loss of consciousness (LOC), headache, vomiting, motor regression, ophthalmoplegia, and visual loss, among others. The complete demographic and patient clinical characteristics are presented in Table 1.

The follow-up duration ranged from 6 to 62 months (mean $29.33 \pm 18.22$ months, median 31 months). During the follow-up period, none of the patients experienced pseudocyst formation or distal shunt malfunction. No adverse event related to the shunt was reported, except for a bilateral chronic subdural hematoma due to shunt overdrainage in case 3.

\section{Illustrative Cases}

\section{Case 3}

A 5-year-old girl presented with neck pain and blurred vision. Brain and cervical MRI revealed hydrocephalus accompanied by tonsillar herniation and a cervical syrinx. On physical examination, the patient had severe papilledema and bilateral sixth cranial nerve paresis. The patient underwent VP shunt placement and her symptoms improved following surgery. After 2 months, the patient was readmitted with worsening vision loss, vomiting, and abdominal distention. Abdominal ultrasound and CT demonstrated a large cystic collection around the clumped distal catheter (Fig. 3). The patient had no history of peritonitis or abdominal surgery. CSF culture was negative. The distal catheter was externalized and the patient received widespectrum antibiotics despite negative cultures from CSF and the tip of the catheter. The patient was scheduled for VP shunt reinsertion after 2 weeks of treatment and cyst resorption on serial ultrasound studies. A new VP shunt on the opposite side of the peritonea resulted in pseudocyst recurrence. The parents of the girl did not agree to VA shunting and a new VP shunt was planned. At surgery, severe peritoneal adhesions forced the surgical team to insert the distal catheter in the suprahepatic space. Her condition improved for several days until she developed repeated headache, vomiting, and drowsiness. A new brain CT scan revealed bilateral subacute subdural hematoma. Burr hole drainage was performed, followed by upgrading the pump to a programmable version and setting it at a higher pressure. The patient improved gradually with no more visual deterioration. After 4 years, she has never experienced another shunt malfunction.

\section{Case 11}

A 14-year-old boy with a history of VP shunting at 3 months of age, and 2 episodes of proximal revisions at the ages of 7 months and 1 year, presented with headache and lethargy 13 years after the last revision. The patient underwent distal shunt revision because of distal catheter detachment (Fig. 4A). He returned to the emergency room after 2 weeks with complaints of ataxic gait, abdominal tenderness, and fluid collection in the thoracic and abdominal shunt track. Physical examination found bilateral papilledema, right-sided ptosis, right-sided sixth cranial 

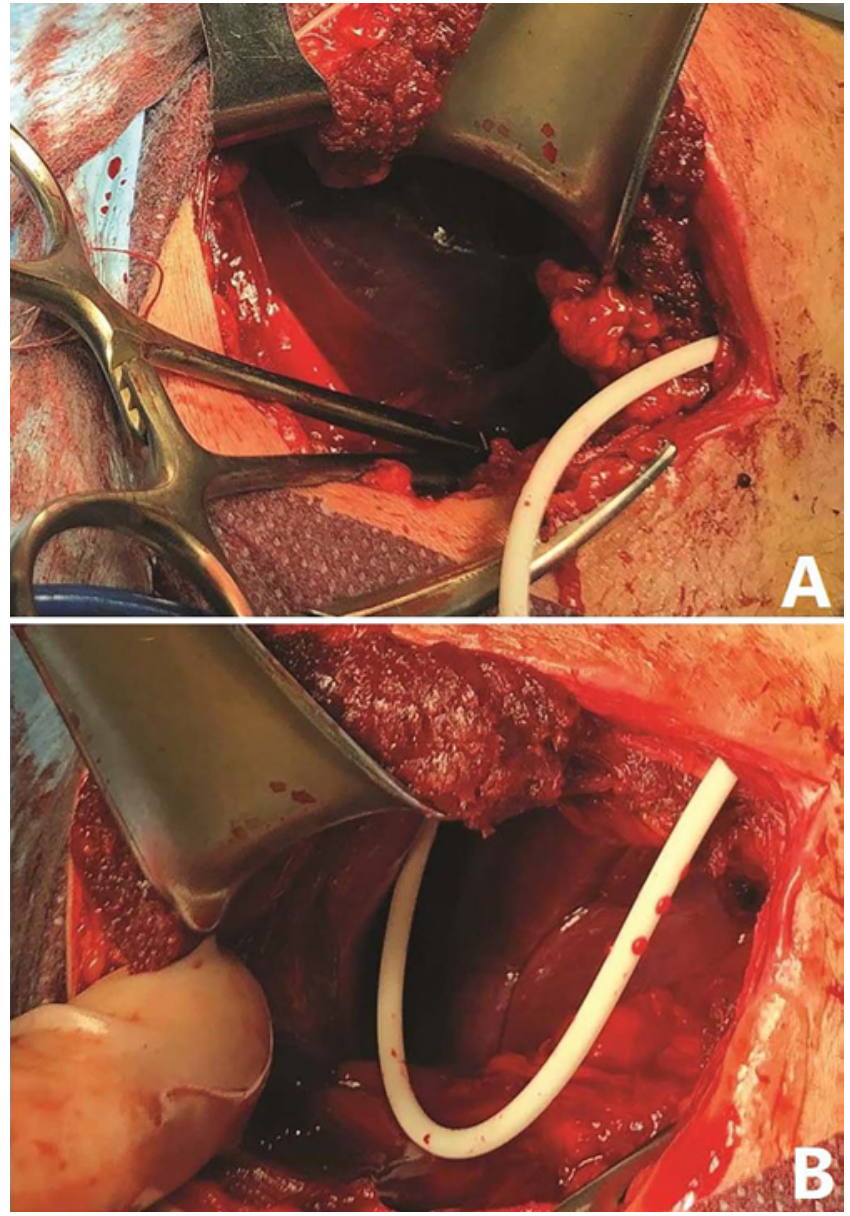

FIG. 2. Surgical view of the suprahepatic space. A: Glossy surface of the liver below the right hemidiaphragm can be seen. B: Distal catheter has been gently slipped and secured over the surface of the liver. Figure is available in color online only.

nerve palsy, and left-sided ophthalmoplegia. Brain CT and MRI revealed no new intracranial lesion, but a large abdominal pseudocyst was found on ultrasound study (Fig. 4B). The patient underwent shunt externalization and antibiotic therapy, followed by serial abdominal ultrasonography and CSF sampling. Again, CSF and distal catheter cultures were negative. After resolving the cyst, a new VP shunt was inserted, resulting in a second pseudocyst formation (Fig. 4C). He underwent a VA shunt placement that failed after a few weeks, and presented with headache followed by seizure and LOC. After temporary CSF diversion, with normal CSF analysis and culture, the patient was scheduled to undergo suprahepatic catheter insertion. He improved postoperatively, and no pseudocyst formed during the 9 months of follow-up. Abdominal plain radiographs, obtained 9 months after surgery, revealed that the distal catheter has remained in the suprahepatic space (Fig. 4D).

\section{Discussion}

Many adverse events associated with VP shunting are reported in the literature, with an incidence rate as high as $47 \%$. Of these, abdominal complications have a rate of approximately $25 \% .^{6}$ In a study by Pan, of 137 patients with a VP shunt, a total of 37 shunt revision surgeries were conducted within 10 years. Abdominal pseudocysts were the cause of approximately $13.5 \%$ of shunt revisions in this series. Four of 5 pseudocysts were discovered after more than 24 months from the last surgery, and 1 was discovered after more than 12 months, signifying the late-onset nature of this complication. ${ }^{7}$ In the current series, peritoneal pseudocyst was detected even 13 years after the last shunt surgery.

The exact pathophysiology of an abdominal pseudocyst is still unclear. It is hypothesized that cyst formation results from an inflammatory reaction to a focal peritonitis or low-grade infection, which further blocks CSF absorption. ${ }^{8}$ Nonetheless, in most instances patients do not show clinical evidence of infection. In this series, only 3 cases had positive CSF culture, even though CSF was kept in culture for only 3 days so slow-growing organisms would be overlooked. The role of slow-growing organisms such as Propionibacterium acnes in distal catheter infection and pseudocyst formation has been discussed in the literature. ${ }^{9}$ Accordingly, it is recommended to add anaerobic cultures and prolonged incubation times of CSF and the distal catheter to detect more infections. Even with negative cultures, this condition is approached therapeutically as a distal infection in most centers. In this series, patients with negative CSF culture received empirical antibiotics for at least 2 weeks. Two cases with gram-negative infection were administrated appropriate antibiotics for 3 weeks, and the patient with a positive culture for Candida underwent antifungal therapy for 8 weeks. A new shunting procedure was not performed until the completion of antibiotic therapy and after obtaining at least 3 consecutive clear CSF samples. Previous abdominal surgeries and intraperitoneal manipulations are proposed as other potential predisposing factors for pseudocyst formation. However, in the current series, only 1 infant (case 8) had a history of surgery for bowel perforation due to NEC.

The main problem encountered with pseudocysts is the relatively high rate of recurrence after treatment. The proposed cause of recurrence is CSF malabsorption due to fibrotic adhesions and lack of epithelial lining subsequent to inflammatory reactions. In a case series by Dzongowski et al., a recurrence rate of $27 \%$ after fluid drainage and shunt revision was reported. ${ }^{3}$ Mobley et al..$^{10}$ described 64 cases, including 46 primary and 18 recurrent abdominal pseudocysts. After resolving the potential infection, 28 of these 64 cases underwent shunt repositioning in the peritoneum, of which $43 \%$ recurred. One patient underwent aspiration only, but later experienced recurrence. The rest of the patients were treated with ventriculopleural, VA, and ventriculocystic shunts. Some authors proposed that the distal catheter can be repositioned in a different abdominal quadrant. Even with such an approach, the rate of recurrence is still high. ${ }^{8}$ In our institution, the first shunt revision after resolving the pseudocyst-associated malfunction is routinely performed with catheter placement in the opposite quadrant. Using this protocol, pseudocysts recurred in 9 of 12 patients, from which 3 (cases 2, 5, and 12) experienced more than 2 episodes of pseudocyst re- 


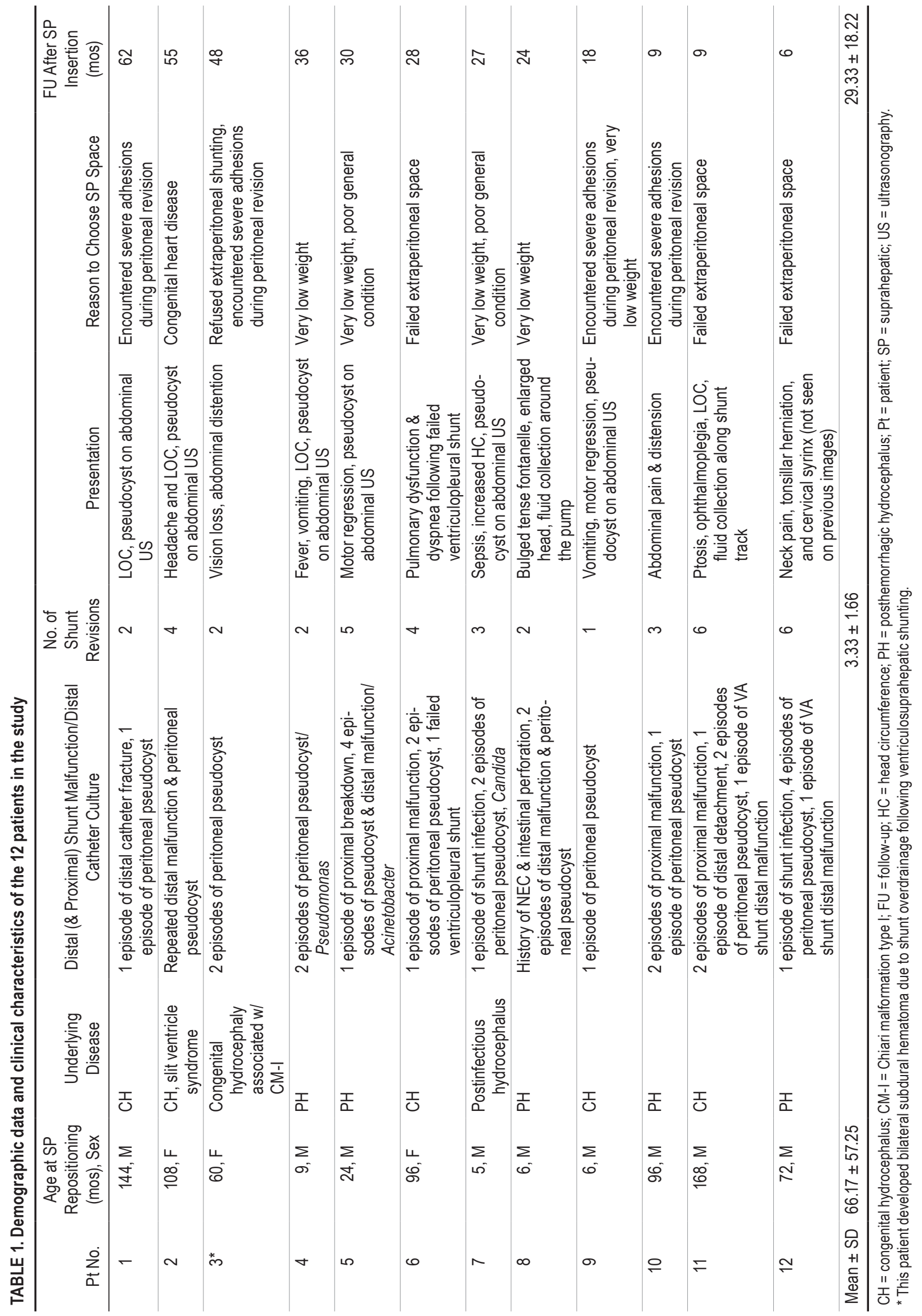




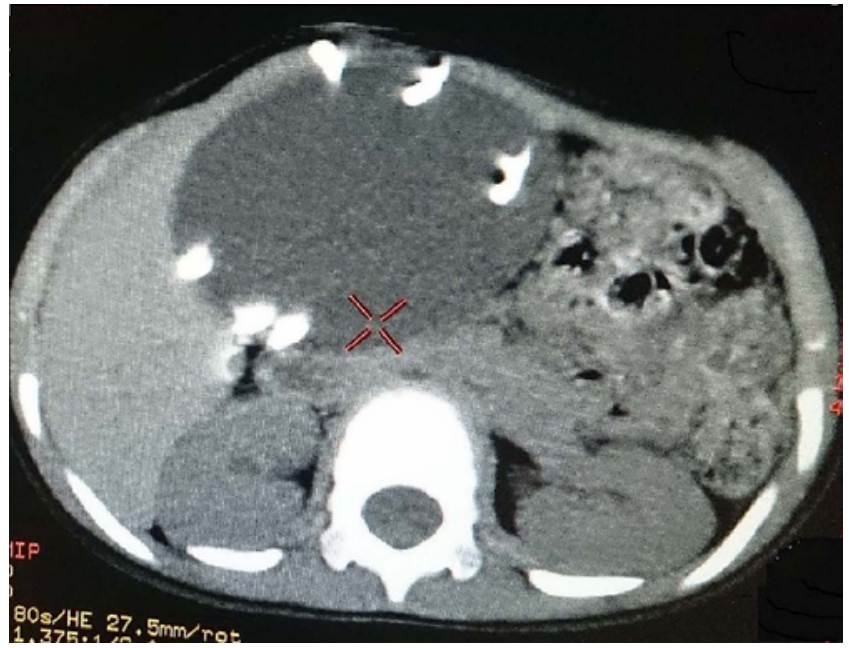

FIG. 3. Case 3. Abdominal CT demonstrating a large cystic collection (red cross) around the clumped distal catheter. Figure is available in color online only.

lapse (Table 1). A potential problematic situation with this approach is encountering severe adhesions when trying to reinsert the catheter on the opposite side. Four cases in the current series (cases 1,3,9, and 10) directly underwent ventriculosuprahepatic shunting at this stage because of encountering such adhesions (Table 1).

Considering the high recurrence rate of this phenomenon, a real challenge is to find a viable space for placement of the distal catheter. In such a situation, ETV can be an option, but ETV is not applicable in all of these complicated cases and many of them require reimplantation of CSF diversion systems. Few alternative spaces such as pleural or atrial cavities are available for distal drainage. Nonetheless, using these spaces may raise technical problems, especially in infants and children. The atrial space has been used as an alternative to the peritoneum for many years. However, substantial morbidity and mortality associated with this method has made it rather unpopular in recent times. ${ }^{11-13}$ Moreover, significant technical difficulties are encountered with placement of atrial catheters in pediatric patients, which further limits the use of this technique. ${ }^{11}$ Congenital heart disease could be an additional obstacle, as was encountered in case 2 .

The pleural space has been never considered as the first choice for distal catheter placement, particularly in pediatric patients. Small pleural surface in this age group may cause pleural effusion. ${ }^{12}$ Hoffman et al. reviewed 59 pediatric patients, including 13 infants, with ventriculopleural shunts. Of these 59 patients, 12 developed pleural effusion, and 6 were under 11 months of age. ${ }^{14}$ In a rare report by Ratliff et al., a ventriculobipleural shunt was inserted as the last resort in a 4-year-old boy who had experienced multiple failures of VP, VA, and ventriculopleural shunts. ${ }^{15}$ This method was proposed to increase the absorbing capacity of the pleural cavity and decrease the potential risk of pleural effusion. ${ }^{15}$

There are sporadic case reports of intractable peritoneal malfunctions managed by ventriculosinus shunting. ${ }^{16,17}$ This is a salvage method in which a distal catheter is placed in the superior sagittal sinus when traditional sites are unavailable. However, the risk of intraoperative bleeding, air embolism, sinus thrombosis, and intracranial hemorrhage should not be overlooked. In recent years, even the iliac crest has been proposed as an alternative receptive site besides the atrium, pleura, bladder, gallbladder, and superior
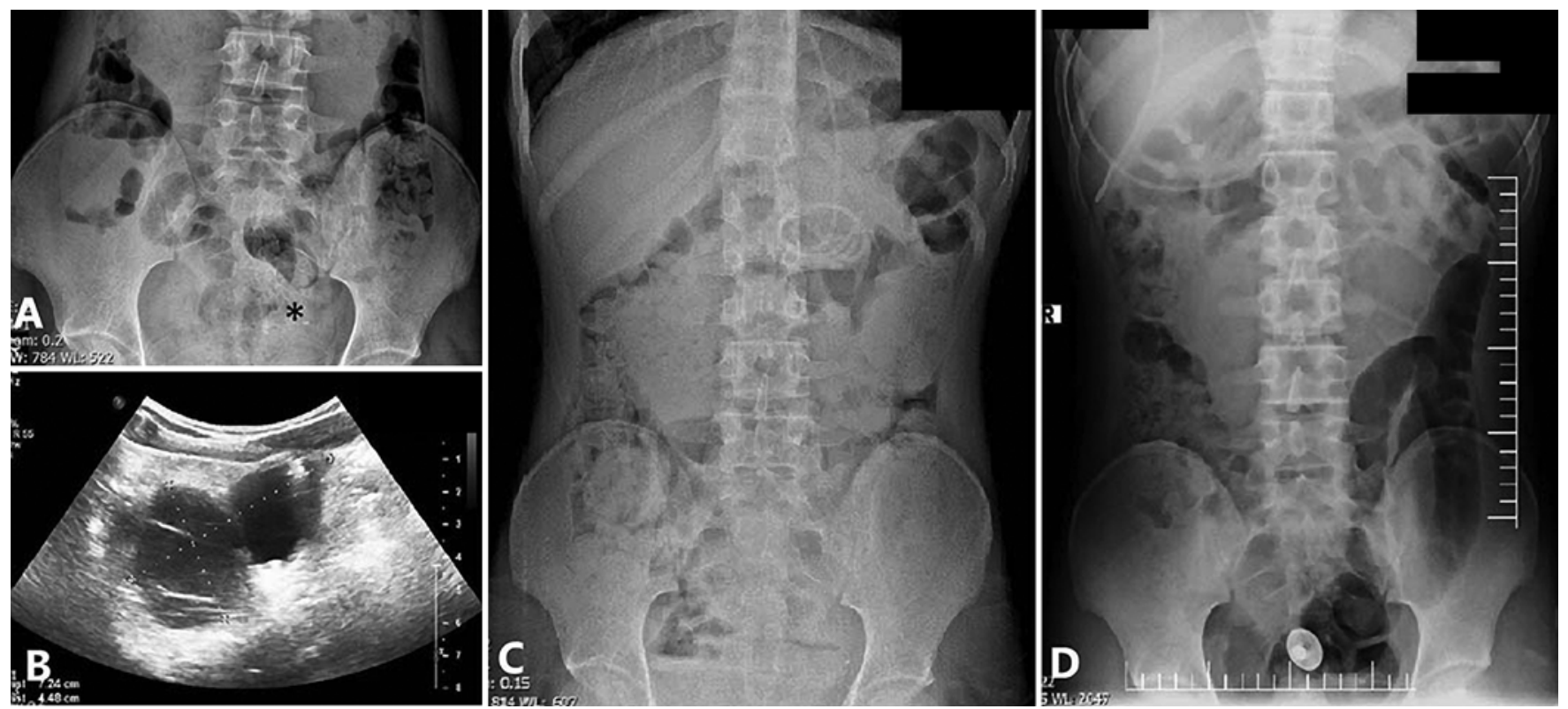

FIG. 4. Case 11. A: Abdominal plain radiograph showing the detached distal catheter (asterisk) in the pelvis. B: Abdominal ultrasonography demonstrates a large abdominal pseudocyst. C: Abdominal plain radiograph shows the clumped distal catheter due to pseudocyst formation. D: Plain radiograph after ventriculosuprahepatic shunting demonstrating the distal catheter in the suprahepatic space. 
sagittal sinus. ${ }^{18}$ In complicated cases of pseudocyst-associated shunt malfunction, neurosurgeons try to find a site for optimal absorption at the expense of more difficult technical approaches or more potential adverse effects.

In this paper, we studied 12 pediatric patients with pseudocyst-associated shunt malfunction who underwent distal catheter placement in the suprahepatic space. Our patients tolerated the surgery well and experienced no adverse events related to shunt malfunction. None of the patients experienced pseudocyst recurrence or distal complications after placing a catheter in the suprahepatic space. In a study conducted by Pandey et al. ${ }^{4}$ on infants undergoing their first shunting procedure, the distal catheter was placed in the suprahepatic space in 133 patients, compared with the general peritoneal cavity in 175 patients. After a mean follow-up of $3.75 \pm 1.45$ years, only 4 complications were observed in the suprahepatic group, 2 of which were pseudocyst formation. In the classic VP shunt group, 55 complications, including 5 pseudocyst formations, occurred within a mean follow-up duration of $4.34 \pm 0.89$ years. The authors concluded that the suprahepatic space could be advantageous over the general peritoneal cavity in terms of lower distal shunt catheter complications. ${ }^{4}$ However, this method did not gain popularity among neurosurgeons for primary shunt insertion. Few other papers could be found regarding use of this approach in patients with peritoneal complications. Rowland et al. reported an adult patient with congenital hydrocephalus who had undergone 25 previous failed CSF diversion procedures and then underwent suprahepatic shunt insertion; the patient had no more reported complications in the 2 years of follow-up. ${ }^{5}$ Yang et al. reported an unusual case of bladder perforation and catheter extrusion through the urethra orifice in a young male patient. A revision surgery was performed and the distal end was fixed in the suprahepatic space but no follow-up was provided. ${ }^{19}$ Rengachary reported a complicated adult case of multiple shunt surgeries in which the patient underwent suprahepatic catheter placement via thoracotomy and transdiaphragmatic conversion of a ventriculopleural to VP shunt. The patient developed no complications after 2 years of follow-up. ${ }^{20}$

In contrast, there are few reports regarding complications of catheter insertion in the suprahepatic space. Adeolu et al. reported pleural effusion as a complication of suprahepatic shunt placement in an 8-year-old patient. It was corrected after revision with shunt placement in the general peritoneal cavity. ${ }^{21}$

Theoretically, the rate of adhesions is lower in the suprahepatic space, ${ }^{4}$ so it might be a good alternative space in cases with several distal malfunctions due to pseudocyst formation in the general peritoneal cavity. This lower rate might be attributable to the lack of mesentery and omentum in the suprahepatic space. Omental clogging is considered a main cause of peritoneal pseudocyst formation following surgeries or inflammatory conditions. As the suprahepatic area is a spared space with no omentum, the chance of pseudocyst formation is hypothetically lower in this space. Moreover, the approach to the suprahepatic space might be technically more feasible in pediatric cases compared with the atrium, pleural cavity, sagittal sinus, or ilium.
Our suggestion is that the suprahepatic space can be a safe alternative site for distal end insertion in those with a history of peritoneal problems. In contrast to Pandey et al., we do not recommend this space for routine VP shunting, as the standard method is more familiar and quicker with no need to extend the laparotomy incision. This approach should be reserved for challenging cases in whom an extra site is needed for distal catheter placement. The role of the pediatric surgeon has to be appreciated for release of the adhesions and accurate placement of the distal catheter. Manipulation of the abdominal cavity after multiple surgeries may harbor the added risk of injury to abdominal viscera, even in the hands of a pediatric surgeon. The role of laparoscopy-assisted placement of the distal catheter in the suprahepatic space and its safety compared to the current method of laparotomy could be examined in further investigations.

To the best of or knowledge, this is the first study to evaluate suprahepatic shunt insertion in a pediatric series with pseudocyst-associated shunt failure. This series does not include all pseudocyst-associated distal malfunctions that underwent revision surgeries in our center, but only those with serious difficulty in peritoneal catheter reinsertion or those having no alternative space for catheter placement. Four patients, including 1 whose parents refused extraperitoneal shunting, were initially candidates for placing the catheter in the opposite side of the peritonea, but the plan was changed during surgery because of severe adhesions in the general perineal cavity. Three infants were selected for this approach after the second peritoneal failure because of their small bodies, rendering them inappropriate candidates for extraperitoneal shunting. One other infant (case 9) was among those who underwent suprahepatic placement during the first revision due to severe adhesions. One patient with cachexia and poor general condition and the other with cor pulmonale were not suitable candidates for extraperitoneal shunting. Suprahepatic shunting was selected in 3 other cases for failed extraperitoneal shunts.

This study is limited by its retrospective nature, small sample size, and lack of a control group. Nonetheless, the purpose of this study was not to compare the suprahepatic space and other available cavities for distal catheter placement. As discussed earlier and shown in the diagram (Fig. 1), suprahepatic shunting is reserved as the last resort for patients who are inappropriate for other approaches. Overall short follow-up duration was another limitation of the study. More prospective trials with larger sample sizes and longer follow-up durations are needed to better evaluate this technique and its long-term results. The other limitation is lack of prolonged incubation time, which could detect more cases of positive cultures, although there would not be much change in surgical management.

\section{Conclusions}

Suprahepatic distal catheter placement after shunt malfunction due to a peritoneal pseudocyst is a safe option. Placement of the peritoneal catheter in the suprahepatic space is not a new concept. It has been previously described, but the current paper suggests it as a last resort in 
challenging situations in which no appropriate extraperitoneal site could be found for safe placement of a distal catheter. Remaining adhesion-free despite severe adhesions in the general peritonea makes the suprahepatic space a potential option for distal catheter reinsertion in cases of severe abdominal adhesions or recurrent pseudocyst formation. The adhesion-free nature of the suprahepatic space is a substantial issue, requiring further investigations. More prospective controlled trials are needed to better evaluate this technique.

\section{References}

1. Kestle JR. Pediatric hydrocephalus: current management. Neurol Clin. 2003;21(4):883-895, vii.

2. Hanlo PW, Cinalli G, Vandertop WP, et al. Treatment of hydrocephalus determined by the European Orbis Sigma Valve II survey: a multicenter prospective 5-year shunt survival study in children and adults in whom a flow-regulating shunt was used. J Neurosurg. 2003;99(1):52-57.

3. Dzongowski E, Coriolano K, de Ribaupierre S, Jones SA. Treatment of abdominal pseudocysts and associated ventricuoperitoneal shunt failure. Childs Nerv Syst. 2017;33(12):2087-2093.

4. Pandey A, Gangopadhyay AN, Sharma SP, et al. Placement of the peritoneal end of a ventriculoperitoneal shunt in the suprahepatic space: does it improve prognosis? Pediatr Neurosurg. 2009;45(1):6-10.

5. Rowland NC, Huang MC, Lawton MT, et al. Treatment of recurrent, multiloculated, obstructive hydrocephalus with suprahepatic peritoneal catheter placement. J Clin Neurosci. 2013;20(7):1038-1039.

6. Guillén A, Costa JM, Castelló I, et al. Unusual abdominal complication of ventriculoperitoneal shunt. Article in Spanish. Neurocirugia (Astur). 2002;13(5):401-404.

7. Pan P. Outcome analysis of ventriculoperitoneal shunt surgery in pediatric hydrocephalus. J Pediatr Neurosci. 2018;13(2):176-181.

8. Yuh SJ, Vassilyadi M. Management of abdominal pseudocyst in shunt-dependent hydrocephalus. Surg Neurol Int. 2012;3:146.

9. Arnell K, Cesarini K, Lagerqvist-Widh A, et al. Cerebrospinal fluid shunt infections in children over a 13 -year period: anaerobic cultures and comparison of clinical signs of infection with Propionibacterium acnes and with other bacteria. $J$ Neurosurg Pediatr. 2008;1(5):366-372.

10. Mobley LW III, Doran SE, Hellbusch LC. Abdominal pseudocyst: predisposing factors and treatment algorithm. Pediatr Neurosurg. 2005;41(2):77-83.

11. Erdoğan H, Altun A, Kuruoğlu E, et al. Difficulties of distal catheter insertion of ventriculoatrial shunting in infants and little children. Turk Neurosurg. 2018;28(4):663-666.
12. Küpeli E, Yilmaz C, Akçay S. Pleural effusion following ventriculopleural shunt: case reports and review of the literature. Ann Thorac Med. 2010;5(3):166-170.

13. Sanders DY, Summers R, DeRouen L. Symptomatic pleural collection of cerebrospinal fluid caused by a ventriculopleural shunt. South Med J. 1997;90(3):345-346.

14. Hoffman HJ, Hendrick EB, Humphreys RP. Experience with ventriculo-pleural shunts. Childs Brain. 1983;10(6):404-413.

15. Ratliff M, Unterberg A, Bächli H. Ventriculo-bipleural shunt as last resort in a 4-year-old child in whom a VP and VA shunt failed. J Neurosurg Pediatr. 2016;17(3):285-288.

16. Oliveira MF, Teixeira MJ, Reis RC, et al. Failed ventriculoperitoneal shunt: is retrograde ventriculosinus shunt a reliable option? World Neurosurg. 2016;92:445-453.

17. Toma AK, Tarnaris A, Kitchen ND, Watkins LD. Ventriculosinus shunt. Neurosurg Rev. 2010;33(2):147-153.

18. Goldstein HE, Feldstein NA, Anderson RC. Ventriculoiliac shunt: a single case experience. J Neurosurg Pediatr. 2015;16(4):477-478.

19. Yang X, Liang R, Zhang Y. An unusual complication of ventriculoperitoneal shunt with bladder perforation and extrusion through the urethra orifice in an adult male patient. Int $J$ Neurosci. 2019;129(1):101-102.

20. Rengachary SS. Transdiaphragmatic ventriculoperitoneal shunting: technical case report. Neurosurgery. 1997;41(3):695-698.

21. Adeolu AA, Komolafe EO, Abiodun AA, Adetiloye VA. Symptomatic pleural effusion without intrathoracic migration of ventriculoperitoneal shunt catheter. Childs Nerv Syst. 2006;22(2):186-188.

\section{Disclosures}

The authors report no conflict of interest concerning the materials or methods used in this study or the findings specified in this paper.

\section{Author Contributions}

Conception and design: Habibi. Acquisition of data: Habibi. Analysis and interpretation of data: Habibi. Drafting the article: Golpayegani. Critically revising the article: Habibi, Tayebi Meybodi. Reviewed submitted version of manuscript: Nejat. Administrative/technical/material support: Ashjaei. Study supervision: Nejat.

\section{Correspondence}

Zohreh Habibi: Children's Medical Center, Tehran University of Medical Sciences, Tehran, Iran.z-habibi@sina.tums.ac.ir. 